\title{
Prevalência de Manifestações Bucais em Pacientes com Câncer Assistidos em um Programa de Atenção Domiciliar na Cidade de Pelotas-RS
}

doi: https://doi.org/10.32635/2176-9745.RBC.2021v67n2.1184

\author{
Prevalence of Oral Manifestations in Cancer Patients Assisted in a Home Care Program in the City of Pelotas-RS \\ Prevalencia de Manifestaciones Orales en Pacientes com Câncer Asistidos en un Programa de Atención Domiciliaria en \\ la Ciudad de Pelotas-RS
}

Bernardo da Fonseca Orcina ${ }^{1}$; Cleusa Marfiza Guimarães Jaccottet ${ }^{2}$; Mônica Cristina Bogoni Savian³

RESUMO

Introdução: O município de Pelotas-RS dispóe do Programa de Internação Domiciliar Interdisciplinar (PIDI) implantado no Hospital Escola da Universidade Federal de Pelotas com a presença de cirurgióes-dentistas em sua equipe. O PIDI atende a pacientes oncológicos em cuidados paliativos. Objetivo: Avaliar a prevalência de manifestaçôes bucais em pacientes assistidos por esse programa, as características epidemiológicas da referida população e verificar a associação das manifestaçôes com o tipo de câncer e tratamento antineoplásico recebido. Método: Estudo transversal retrospectivo, com dados secundários coletados dos prontuários odontológicos de pacientes atendidos no período de abril de 2018 a setembro de 2019. O teste qui-quadrado foi aplicado com um nível de $5 \%$ de significância ( $\mathrm{p} \leq 0,05)$ atribuído aos resultados significativos. Resultados: As manifestaçóes bucais foram encontradas 47 vezes nos 61 pacientes avaliados, sendo que alguns apresentaram mais de uma ocorrência. Pacientes que apresentavam câncer primário no sistema digestivo foram os mais acometidos por manifestaçôes bucais. As mais prevalentes foram xerostomia (26,2\%); candidíase (18\%); mucosite (13,1\%); disfagia e disgeusia, que apresentaram o mesmo percentual (9,8\%); nenhum paciente desenvolveu cárie de radiação. A xerostomia mostrou significância estatística $(\mathrm{p} \leq 0,05)$ na associação com o tipo de câncer. Ao correlacionar as manifestaçóes bucais com o tipo de tratamento recebido, estas não apresentaram significância estatística. Conclusão: Este estudo demonstrou alta prevalência de manifestaçóes bucais na população estudada, reafirmando a importância do cirurgião-dentista na equipe multiprofissional de cuidados paliativos.

Palavras-chave: Odontologia; Odontólogos; Cuidados Paliativos; Oncologia; Assistência Domiciliar.

\section{ABSTRACT}

Introduction: The municipality of Pelotas-RS has the Interdisciplinary Home Internment Program (PIDI) implemented at the Teaching Hospital of the Federal University of Pelotas with the presence of dental surgeons on its team. PIDI assists oncology patients in palliative care. Objective: To evaluate the prevalence of oral manifestations in patients assisted by this program, the epidemiological characteristics of that population and to verify the relationship of the manifestations with the type of cancer and antineoplastic treatment received. Method: Retrospective cross-sectional study with secondary data collected from the dental records of patients seen from April 2018 to September 2019. The Chi-square test was applied, with a $5 \%$ significance level $(\mathrm{p} \leq 0.05)$ attributed to significant results. Results: Oral manifestations were found 47 times in the 61 patients evaluated, with some having more than one occurrence. Patients who had primary cancer in the digestive system were the most affected by oral manifestations. The most prevalent oral manifestations were xerostomia (26.2\%), followed by candidiasis $(18 \%)$, mucositis $(13.1 \%)$, dysphagia and dysgeusia that presented the same percentage $(9.8 \%)$, no patient developed radiation caries. Xerostomia showed statistical significance $(\mathrm{p} \leq 0.05)$ in relation to the type of cancer. When correlating oral manifestations with the type of treatment received, they were not statistically significant. Conclusion: This study demonstrated a high prevalence of oral manifestations in the population investigated, reaffirming the importance of the dental surgeon in the palliative care multidisciplinary team.

Key words: Dentistry; Dentists; Palliative Care; Medical Oncology; Home Nursing.
RESUMEN

Introducción: El municipio de Pelotas-RS cuenta con el Programa Interdisciplinario de Internación Domiciliaria (PIDI) implementado en el Hospital Docente de la Universidad Federal de Pelotas con la presencia de cirujanos dentistas en su equipo. PIDI atiende a pacientes oncológicos en cuidados paliativos. Objetivo: Evaluar la prevalencia de manifestaciones bucales en pacientes atendidos por este programa, las características epidemiológicas de esa población y verificar la relación de las manifestaciones con el tipo de cáncer y tratamiento antineoplásico recibido. Método: Estudio transversal retrospectivo con datos secundarios recolectados de los registros dentales de los pacientes atendidos desde abril de 2018 a septiembre de 2019. Se aplicó la prueba de chi-cuadrado, con un nivel de significancia del $5 \%(\mathrm{p} \leq 0,05)$ atribuido a resultados significativos. Resultados: Las manifestaciones orales se encontraron 47 veces en los 61 pacientes evaluados, algunos con más de una ocurrencia. Los pacientes que tenían cáncer primario en el sistema digestivo fueron los más afectados por las manifestaciones orales. Las más prevalentes fueron xerostomía (26,2\%); candidiasis (18\%); mucositis (13,1\%); disfagia y disgeusia, que presentaron el mismo porcentaje $(9,8 \%)$; ningún paciente desarrolló caries de radiación. La xerostomía mostró significancia estadística $(\mathrm{p} \leq 0,05)$ en relación con el tipo de cáncer. Al correlacionar las manifestaciones orales con el tipo de tratamiento recibido, no resultaron estadísticamente significativas. Conclusión: Este estudio demostró una alta prevalencia de manifestaciones bucales en la población estudiada, reafirmando la importancia del cirujano dentista en el equipo multidisciplinario de cuidados paliativos.

Palabras clave: Odontología; Odontólogos; Cuidados Paliativos; Oncología Médica; Atención Domiciliaria de Salud.

\footnotetext{
1,2,3 Hospital Escola da Universidade Federal de Pelotas (UFPel). Pelotas (RS), Brasil.

'E-mail: bernardoforcina@outlook.com. Orcid iD: https://orcid.org/0000-0003-3367-483X

2E-mail: cleusajaccottet@hotmail.com. Orcid iD: https://orcid.org/0000-0003-2413-871X

${ }^{3}$ E-mail: monica.savian@ebserh.gov.br. Orcid iD: https://orcid.org/0000-0002-1428-330X
}

Endereço para correspondência: Bernardo da Fonseca Orcina. Praça São Marcos, 36 - Laranjal. Pelotas (RS), Brasil. CEP 96090-720. E-mail: bernardoforcina@outlook.com 


\section{INTRODUÇÃO}

Com relação ao câncer no Brasil, a estimativa de novos casos para o triênio 2020-2022 é de 625 mil em cada um dos anos, sendo que mais de $80 \%$ deles seriam registrados nas Regiôes Sul e Sudeste ${ }^{1}$. Essa perspectiva de aumento da incidência do câncer reflete o crescimento da população idosa, bem como alteraçôes na prevalência e distribuição dos principais fatores de risco, sendo vários destes ligados ao desenvolvimento socioeconômico ${ }^{2}$.

Os cuidados paliativos (CP) em odontologia podem ser definidos como o manejo de pacientes com doença avançada ou progressiva, cuja cavidade oral pode ser comprometida pela doença de base, por doença sistêmica ou pelo seu tratamento, sendo o foco do atendimento a melhora da qualidade de vida ${ }^{3}$.

O município de Pelotas dispóe do Programa de Internação Domiciliar Interdisciplinar (PIDI), implantado em 2005 no Hospital Escola da Universidade Federal de Pelotas (HE/UFpel). O Programa conta com equipes multiprofissionais de referência compostas por um profissional da área médica e um da equipe de enfermagem que realizam duas visitas ao dia; e uma equipe de apoio que realiza visitas semanais composta por nutricionistas, psicólogos, terapeutas ocupacionais, assistentes sociais, fisioterapeutas e cirurgiōes-dentistas ${ }^{4}$.

Os desconfortos bucais estão entre os sintomas mais comuns do paciente em CP, sendo que estes aumentam à medida que a doença de base evolui, evidenciando que a cavidade bucal geralmente é o primeiro local de desconforto e perda de função $0^{5,6}$. Com essa progressão, algumas alteraçóes como candidíase, mucosite, xerostomia, disfagia, disgeusia e cárie de radiação podem ser vivenciadas pelos pacientes oncológicos em final de vida ${ }^{3,5,7}$.

A candidíase pode atingir de $10 \%$ a $57 \%$ dos pacientes com câncer ${ }^{3,5}$. Já a mucosite está presente em quase a totalidade dos pacientes sob terapia radioterápica de cabeça e pescoço, entre $75 \%$ a $100 \%$ dos indivíduos que realizam transplante de células hematopoiéticas e em cerca de $40 \%$ dos pacientes que são submetidos a protocolos quimioterápicos específicos ${ }^{8-12}$. A prevalência de xerostomia na população geral varia de 5,5\% e pode alcançar 46\%, mostrando variaçóes de acordo com o sexo, idade e outros fatores associados ${ }^{13}$. Alguns estudos com pacientes com câncer em final de vida apontam que essa prevalência pode chegar até $78 \%$ ou $81 \%$, Anteriormente aos tratamentos antineoplásicos, a prevalência de disfagia, conceituada como a dificuldade em conduzir os alimentos para deglutição, pode ser de $21 \%$, enquanto aumenta para $28 \%$ ao término destes ${ }^{14}$. Em pacientes oncológicos em $\mathrm{CP}$, a disgeusia, caracterizada como a alteração na percepçáo no paladar, pode ser encontrada em $25 \%$ a $50 \%$ deles $^{15}$. E naqueles que recebem tratamento radioterápico de cabeça e pescoço, a incidência de cárie pós-radiação de cabeça e pescoço é de $29 \%$, podendo chegar a aproximadamente $37 \%$ dos casos após dois anos do término do tratamento ${ }^{16}$. As manifestaçóes bucais em pacientes com câncer têm sido tema de diversos estudos, entretanto, julga-se importante estudar a prevalência dessas manifestaçóes em pacientes com câncer em cuidados paliativos atendidos na atenção domiciliar, visando a contribuir para o melhor entendimento desse tema e para o planejamento dos serviços de atenção domiciliar para essa populaçáo.

O objetivo do presente estudo foi avaliar a prevalência das principais manifestações bucais em pacientes com câncer em CP internados no PIDI do HE/UFPel; descrever as características da população segundo sexo e idade; e verificar a possível associação entre a presença das alteraçôes bucais e o tipo de câncer e entre a apresentação de manifestaçóes bucais e o tratamento antineoplásico recebido.

\section{MÉTODO}

Realizou-se um estudo epidemiológico transversal com dados secundários, visando a avaliar a prevalência de manifestaçōes orais em pacientes com câncer em CP atendidos pelo PIDI do Hospital Escola da UFPel. Alguns critérios de elegibilidade são necessários para que o paciente possa ser admitido na internaçáo domiciliar, como: um perfil clínico agudo, crônico agudizado, restrito ao leito, crônico complexo; ou seja, aqueles que têm necessidade de CP no mínimo semanal, com a finalidade de controlar a dor e sofrimento, decorrentes da doença oncológica e seus tratamentos. Os períodos de internação podem ser transitórios, curtos, longos ou definitivos ${ }^{17}$.

A amostra deste estudo foi composta dos pacientes assistidos pela equipe da odontologia durante o período da coleta de dados, que compreendeu os meses de abril de 2018 a setembro de 2019, totalizando 61 participantes.

Os dados sociodemográficos e do histórico clínico foram extraídos dos prontuários do PIDI, sem cegamento de sujeitos, após treinamento prévio do autor. Nos prontuários, constavam informaçóes da equipe multiprofissional relacionadas a aspectos clínicos, odontológicos, psicológicos, espirituais e sociais. Os dados referentes à condição de saúde bucal encontravam-se registrados em um prontuário específico utilizado pelo serviço, contendo informaçóes da anamnese odontológica e do exame clínico, como presença de dores na cavidade bucal, presença e aspecto de lesôes orais, aumento de volume na regiāo de cabeça e pescoço, disfagia, disgeusia, xerostomia e manifestaçóes bucais decorrentes do tratamento antineoplásico, e a necessidade de tratamento 
odontológico. Os pacientes assistidos pelo programa, que apresentaram necessidades de tratamento, são atendidos em domicílio ou encaminhados para atendimento clínico no consultório odontológico do Hospital Escola HE/ UFPel, conforme sua necessidade clínica.

As variáveis analisadas neste estudo foram definidas por serem, segundo dados da literatura, as afecçôes bucais mais prevalentes nos pacientes oncológicos em $\mathrm{CP}^{3,5,7}$. Sendo elas, candidíase oral, mucosite, xerostomia, disfagia, disgeusia e cárie de radiação.

As localizaçôes do câncer nos pacientes internados no programa foram bastante variadas: mama, próstata, boca, laringe, glote, amígdala, estômago, duodeno, esôfago, reto, fígado, vias biliares, pâncreas, pulmôes, útero, ovário, vulva, endométrio, melanoma, bexiga, testículo, rim, mediastino, entre outras. A fim de melhor realizar a análise dos dados e diminuir a variabilidade, os tipos de câncer foram agrupados em grandes grupos, sendo eles: cabeça e pescoço, mama, próstata, sistema digestivo, sistema respiratório, sistema reprodutor feminino e outros.

Os dados obtidos foram tabulados em planilha eletrônica no software Excel (Microsoft, versão 15.0), Albuquerque, Novo México, EUA e avaliados por meio do programa Statistical Package for the Social Sciences (SPSS), Chicago, IL, EUA, versão 17.0.

O teste qui-quadrado foi aplicado com um nível de $5 \%$ de significância $(\mathrm{p} \leq 0,05)$ atribuído aos resultados significativos para verificar a associação entre as manifestaçôes bucais e o tipo de câncer diagnosticado nos pacientes. Também, foi utilizado para observar a associação das afecçóes bucais com o tipo de tratamento antineoplásico recebido. As características epidemiológicas da populaçáo foram realizadas de maneira descritiva.

O trabalho foi aprovado pelo Comitê de Ética em Pesquisa da Faculdade de Medicina da Universidade Federal de Pelotas sob o número de parecer 3.372.405.

\section{RESULTADOS}

A amostra foi composta de 61 pacientes, sendo 27 $(44,3 \%)$ do gênero feminino e $34(55,7 \%)$ do masculino. A idade variou entre 27 e 89 anos, com uma mediana de 64 anos, média de 63,9 anos, e desvio-padráo de 14,4 anos.

Em relação aos tratamentos antineoplásicos recebidos pelos pacientes, oito (13\%) receberam tratamento radioterápico na região de cabeça e pescoço e 37 $(60,7 \%)$ tiveram a conduta terapêutica de infusão de quimioterápico. No total, sete $(11,5 \%)$ dos pacientes receberam tanto o tratamento quimioterápico quanto a radioterapia de cabeça e pescoço.

As manifestaçôes bucais tiveram presentes por 47 vezes em 35 pacientes (57,3\%), sendo que, em 11 (18\%) deles, o diagnóstico de duas dessas variáveis esteve presente. $\mathrm{Na}$ Tabela 1, observa-se a prevalência de cada manifestação bucal.

Tabela 1. Prevalência das manifestações bucais

\begin{tabular}{lcc}
\hline Manifestações bucais & $\mathbf{n}$ & $\%$ \\
\hline Candidíase & 11 & 18 \\
Mucosite & 8 & 13,1 \\
Xerostomia & 16 & 26,2 \\
Disfagia & 6 & 9,8 \\
Disgeusia & 6 & 9,8 \\
Cárie de radiação & 0 & 0 \\
\hline
\end{tabular}

Da amostra desta pesquisa, o local de câncer mais prevalente foi o sistema digestivo, manifestando-se em $22(36,1 \%)$ indivíduos, seguido por cabeça e pescoço e sistema respiratório em sete $(11,5 \%)$ (Tabela 2$)$.

Tabela 2. Distribuição da prevalência de pacientes por grupos de câncer

\begin{tabular}{lcc}
\hline Local do câncer & $\mathbf{n}$ & $\mathbf{\%}$ \\
\hline Cabeça e pescoço & 7 & 11,5 \\
Mama & 5 & 8,2 \\
Próstata & 5 & 8,2 \\
Sistema digestório & 22 & 36,1 \\
Sistema endócrino & 3 & 4,9 \\
Sistema reprodutor feminino & 6 & 9,8 \\
Sistema respiratório & 7 & 11,5 \\
Outros & 5 & 8,2 \\
Sem informação & 1 & 1,6 \\
\hline TOTAL & $\mathbf{6 1}$ & $\mathbf{1 0 0 , 0}$ \\
\hline
\end{tabular}

Os pacientes mais acometidos por alteraçóes bucais apresentavam a localização do câncer nos seguintes grupos: sistema digestivo, cabeça e pescoço e próstata, sendo estes dois últimos com o mesmo número de manifestaçóes. As distribuiçôes das manifestações nos grupos são observadas na Tabela 3.

Ao relacionar as manifestaçóes bucais e o local do câncer, apenas a xerostomia mostrou-se significativa ( $\mathrm{p}$ 0,030) ao nível de 5\% de significância (Tabela 3).

Cada manifestação bucal foi testada estatisticamente por meio do teste qui-quadrado com o objetivo de verificar a associação entre o tratamento radioterápico na regiāo de cabeça e pescoço e quimioterápico. Assim, destaca-se, neste estudo, que não foi encontrada associação significativa entre os tratamentos antineoplásicos e as afecções bucais (ao nível de 5\%). 
Tabela 3. Manifestação bucal distribuída conforme o local de câncer e resultado da associação estatística

\begin{tabular}{|c|c|c|c|c|c|c|c|c|c|c|c|c|c|c|c|}
\hline \multirow[b]{3}{*}{ Local do câncer } & \multicolumn{15}{|c|}{ Manifestações bucais } \\
\hline & \multicolumn{3}{|c|}{ Xerostomia } & \multicolumn{3}{|c|}{ Disfagia } & \multicolumn{3}{|c|}{ Disgeusia } & \multicolumn{3}{|c|}{ Candidíase } & \multicolumn{3}{|c|}{ Mucosite } \\
\hline & n & $\%$ & p-valor & n & $\%$ & p-valor & $\mathbf{n}$ & $\%$ & p-valor & $\mathbf{n}$ & $\%$ & p-valor & n & $\%$ & p-valor \\
\hline Cabeça e pescoço & 3 & 18,8 & & 1 & 16,7 & & 1 & 16,7 & & 1 & 9,1 & & 0 & 0,0 & \\
\hline Mama & 2 & 12,5 & & 0 & 0,0 & & 0 & 0,0 & & 0 & 0,0 & & 0 & 0,0 & \\
\hline Próstata & 2 & 12,5 & & 1 & 16,7 & & 1 & 16,7 & & 1 & 9,1 & & 1 & 12,5 & \\
\hline Sistema digestivo & 5 & 31,3 & & 2 & 33,3 & & 2 & 33,3 & & 5 & 45,5 & & 6 & 75,0 & \\
\hline Sistema endócrino & 0 & 0,0 & $0,030^{*}$ & 1 & 16,7 & 0,108 & 0 & 0,0 & 0,140 & 2 & 18,2 & 0,274 & 0 & 0,0 & 0,238 \\
\hline $\begin{array}{l}\text { Sistema reprodutor } \\
\text { feminino }\end{array}$ & 2 & 12,5 & & 0 & 0,0 & & 1 & 16,7 & & 0 & 0,0 & & 0 & 0,0 & \\
\hline Sistema respiratório & 1 & 6,3 & & 1 & 16,7 & & 1 & 16,7 & & 1 & 9,1 & & 1 & 12,5 & \\
\hline Outros & 0 & 0,0 & & 0 & 0,0 & & 0 & 0,0 & & 1 & 9,1 & & 0 & 0,0 & \\
\hline Sem informação & 1 & 6,3 & & 0 & 0,0 & & 0 & 0,0 & & 0 & 0,0 & & 0 & 0,0 & \\
\hline TOTAL & 16 & 100,0 & & 6 & 100,0 & & 6 & 100,0 & & 11 & 100,0 & & 8 & 100,0 & \\
\hline
\end{tabular}

Legenda: *Nível de 5\% de significância.

\section{DISCUSSÃO}

No presente estudo, foram avaliados pacientes em CP, sendo a localização de câncer mais prevalente o sistema digestivo. Segundo estimativa do Instituto Nacional de Câncer José Alencar Gomes da Silva (INCA) ${ }^{1}$, para o ano de 2020, excetuando o câncer de pele náo melanoma, os cânceres de mama e próstata serão os mais incidentes, seguidos de cólon e reto, ambos do sistema digestivo. Os referidos cânceres apresentam uma possibilidade de cura de $75 \%$ dos casos, sendo a taxa relativa de sobrevivência em cinco anos de 90,8\% e 99,5\%, respectivamente, o que pode explicar a maior prevalência do câncer do aparelho digestivo na população estudada, visto que a amostra foi composta por pacientes em CP com estágio avançado da doenca $^{18}$.

Efeitos adversos aparecem à medida que a doença oncológica avança, estando relacionados tanto aos tratamentos antineoplásicos como às condiçóes sistêmicas. A xerostomia é a manifestação mais comum encontrada nos pacientes em CP, podendo estar associada a: desidratação, desequilíbrio metabólico, estado geral do paciente, tratamentos antineoplásicos, principalmente radioterapia de cabeça e pescoço, e medicaçóes, pois muitos deles recebem polifarmácia e fazem uso de drogas que têm como efeito colateral a xerostomia ${ }^{3,5}$, tornando-se a manifestação bucal mais prevalente no presente estudo $(26,2 \%)$, corroborando os dados da literatura ${ }^{13,19}$, além de apresentar associação estatística com o local do câncer.

A cárie de radiaçáo possui uma prevalência de aproximadamente $24 \%$ nos pacientes que realizam radioterapia de cabeça e pescoço e $21 \%$ nos que realizam esse tratamento associado à quimioterapia ${ }^{16}$. Em um estudo realizado na Suécia, foram acompanhados 126 pacientes com câncer de cabeça e pescoço durante dez anos. Observou-se que, antes do tratamento radioterápico, 23 dentes desses indivíduos necessitavam tratamento restaurador e que, após a radiação, esse número aumentou para 281 dentes em 67 pacientes $^{20}$. No presente estudo, nenhum paciente apresentou cárie de radiaçáo, o que pode ter ocorrido pelo tempo de acompanhamento limitado para observar a lesão, como também pelo tamanho amostral, visto que apenas oito pacientes haviam realizado radioterapia na regiâo de cabeça e pescoço.

As manifestaçôes bucais: disfagia, disgeusia, candidíase e mucosite não apresentaram associação com o local do câncer primário. A disfagia esteve presente em $9,8 \%$ da amostra desta pesquisa, alguns autores ${ }^{14}$ evidenciaram uma maior incidência em torno de $21 \%$ no momento do diagnóstico do câncer e $28 \%$ após o tratamento antineoplásico. Antes do tratamento, a disfagia foi associada aos tumores de laringe e hipofaringe, enquanto a disfagia após cirurgia e/ou radioterapia foi relacionada às neoplasias de boca e orofaringe.

A disgeusia apresenta um caráter multifatorial ${ }^{21-24}$, sendo um desses fatores os efeitos colaterais dos tratamentos oncológicos, porém, neste estudo, não foi possível evidenciar relações estatísticas dessa alteração bucal com a radioterapia de cabeça e pescoço e quimioterapia.

Apesar disso, a literatura mostra sua prevalência em $56,3 \%$ dos pacientes tratados com quimioterapia, $66,5 \%$ nos tratados com radioterapia, podendo chegar a $76 \%$ quando associadas essas duas modalidades antineoplásicas ${ }^{25}$. Por outro lado, 9,8\% dos pacientes em final de vida apresentaram tal manifestação nesta pesquisa. Nos pacientes em CP do PIDI, havia aqueles que já tinham 
realizado tratamento quimioterápico antes da internação no programa, o que pode ter justificado a ausência de associação entre a disgeusia e o tratamento antineoplásico. Outro estudo observou equiparação da disgeusia com a quimioterapia, porém todos os pacientes estavam em tratamento quimioterápico no momento da avaliação ${ }^{26}$.

Com relação à candidíase oral, nesta pesquisa foi encontrado um percentual de $18 \%$, o que corrobora a estimativa de $10 \%$ a $40 \%$ encontrada em outros estudos $^{27,28}$, podendo chegar a $57 \% \%^{3}$. Em um estudo ${ }^{29}$ no qual foi realizada a avaliação de 54 pacientes em um hospice da Dinamarca, 48\% deles apresentaram sintomas clínicos de candidíase orofaríngea tratada inadequadamente quando admitidos no hospice, o que foi confirmado com a presença da forma de hifa na análise microscópica. Isto reforça que, quando essa infecção não é tratada de maneira adequada por um profissional capacitado, esses microrganismos podem exibir resistência intrínseca ou adquirida.

A mucosite é caracterizada como uma inflamação dolorosa, frequentemente ulcerada no trato digestivo, pode estar presente em $100 \%$ dos pacientes que recebem radioterapia de cabeça e pescoço e está relacionada com os protocolos quimioterápicos adotados ${ }^{8-12,30}$. Esses dados, muitas vezes, encontram-se ligados a pacientes com câncer, não especificando aqueles em $\mathrm{CP}$. Nesta pesquisa, a mucosite teve uma prevalência de $13,1 \%$; já em estudos ${ }^{7,31}$ em que também foram estudados pacientes em CP, sendo eles em internação domiciliar, hospice ou unidade de CP, os valores apresentaram-se entre $10,4 \%$ e $22,3 \%$. Ainda, no estudo ${ }^{31}$ com maior acometimento, a localizaçáo primária de câncer mais prevalente foi o sistema digestivo, semelhante ao encontrado neste trabalho. Na presente pesquisa, não foram encontradas relaçóes significativas entre a mucosite e o grupo cabeça e pescoço ou com outros tratamentos antineoplásicos, visto que não esteve presente nos pacientes com câncer de cabeça e pescoço avaliados, tendo sua maior prevalência nos pacientes com câncer do sistema digestivo.

A ausência da mucosite em alguns pacientes pode ser explicada por estes terem sido integrados no programa após terem concluído seus tratamentos antineoplásicos, sugerindo reparo das lesóes bucais. Alguns protocolos quimioterápicos para tratamento de câncer de cólon e reto, por exemplo, 5-fluoracil ou capecitabina, podem apresentar durante o tratamento até 50\% dos casos dessa manifestação bucal ${ }^{32}$.

A heterogeneidade do ponto de vista da localizaçáo primária da neoplasia e o tipo de tratamento podem exigir uma ampliação da amostra para que se obtenha a associação entre as afecçóes bucais e o tipo de tratamento antineoplásico recebido. Este estudo apresenta validade interna e demonstrou alta prevalência de manifestaçóes bucais na população estudada, reafirmando a importância do cirurgião-dentista na equipe multiprofissional de CP.

\section{CONCLUSÃO}

A complexidade para atender um paciente em CP exige tanto um amplo conhecimento dos sinais e sintomas que lhe acometem em final de vida quanto vivenciar uma atuação multiprofissional.

A partir deste estudo, foi possível concluir que a maioria dos pacientes com câncer em CP avaliados pela equipe odontológica era do sexo masculino (55,7\%), com uma média de idade de 63,9 anos, e tinha diagnóstico primário no sistema digestivo. A xerostomia foi a manifestação bucal mais prevalente, a presença de mucosite foi maior no grupo dos pacientes que apresentavam lesôes oncológicas no sistema digestivo, em decorrência dos protocolos quimioterápicos. As manifestaçôes bucais disfagia, disgeusia, candidíase e mucosite não apresentaram associação com o tipo de tratamento e localização primária do tumor. A xerostomia foi a manifestação que apresentou associação significativamente estatística com o local do câncer.

O presente estudo buscou contribuir para o conhecimento das principais manifestaçôes bucais em pacientes em CP assistidos em domicílio, visando à melhoria do cuidado e da qualidade de vida.

\section{CONTRIBUIÇÕES}

Todos os autores contribuíram na concepção el ou no planejamento do estudo; na obtençáo, análise e interpretação dos dados; assim como na redação e revisão crítica; e aprovaram a versão final a ser publicada.

\section{DECLARAÇÃO DE CONFLITO DE INTERESSES}

Nada a declarar.

\section{FONTES DE FINANCIAMENTO}

Não há.

\section{REFERÊNCIAS}

1. Instituto Nacional de Câncer José Alencar Gomes da Silva. Estimativa 2020: incidência de câncer no Brasil. Rio de Janeiro: INCA; 2019.

2. Bray F, Ferlay J, Soerjomataram I, et al. Global cancer statistics 2018: GLOBOCAN estimates of incidence and mortality worldwide for 36 cancers in 185 countries. CA Cancer J Clin. 2018;68(6):394-424. doi: https://doi. org/10.3322/caac. 21492 
3. Wiseman M. Palliative care dentistry: focusing on quality of life. Compend Contin Educ Dent. 2017;38(8):52935.

4. Fripp JC, Facchini LA, Silva SM. Caracterização de um programa de internação domiciliar e cuidados paliativos no Município de Pelotas, Estado do Rio Grande do Sul, Brasil: uma contribuição à atenção integral aos usuários com câncer no Sistema Único de Saúde, SUS. Epidemiol Serv Saúde. 2012;21(1):69-78. doi: http:// doi.org/10.5123/S1679-49742012000100007

5. Matsuo K, Watanabe R, Kanamori D, et al. Associations between oral complications and days to death in palliative care patients. Support Care Cancer. 2016;24(1):157-61. doi: http://doi.org/10.1007/s00520-015-2759-9

6. Delgado MB, Burns L, Quinn C, et al. Oral care of palliative care patients - carers' and relatives' experiences. A qualitative study. Br Dent J. 2018;224(11):881-6. doi: http://doi.org/10.1038/sj.bdj.2018.434

7. Nakajima N. Characteristics of oral problems and effects of oral care in terminally ill patients with câncer. Am J Hosp Palliat Care. 2017 Jun;34(5):430-4. Epub 2016 Feb 22. doi: http://doi.org/10.1177/1049909116633063

8. Robien K, Schubert MM, Bruemmer B, et al. Predictors of oral mucositis in patients receiving hematopoietic cell transplants for chronic myelogenous leukemia. J Clin Oncol. 2004;22(7):1268-75. doi: http://doi. org/10.1200/JCO.2004.05.147

9. Sonis ST. The pathobiology of mucositis. Nat Rev Cancer. 2004 Apr;4(4):277-84. doi: http://doi. org/10.1038/nrc1318

10. Elting LS, Cooksley CD, Chambers MS, et al. Risk, outcomes, and costs of radiation-induced oral mucositis among patients with head-and-neck malignancies. Int J Radiat Oncol Biol Phys. 2007;68(4):1110-20. doi: http://doi.org/10.1016/j.ijrobp.2007.01.053

11. Lalla RV, Saunders DP, Peterson DE. Chemotherapy or radiation-induced oral mucositis. Dent Clin North Am. 2014;58(2):341-9. doi: http://doi.org/10.1016/j. cden.2013.12.005

12. Curra M, Soares Junior LAV, Martins MD, et al. Protocolos quimioterápicos e incidência de mucosite bucal. Revisão Integrativa. Einstein (São Paulo). 2018;16(1):1-9. doi: https://doi.org/10.1590/s167945082018 rw 4007

13. Villa A, Connell CL, Abati S. Diagnosis and management of xerostomia and hyposalivation. Ther Clin Risk Manag. 2015;11:45-51. doi: https://doi.org/10.2147/TCRM. S76282

14. Martín Villares C, Tapia Risueño M, San Román Carbajo J, et al. Disfagia pretratamiento en pacientes con cáncer avanzado de cabeza y cuello. Nutr Hosp. 2003;18(5):238-42.

15. Mirza N, Machtay M, Devine PA, et al. Gustatory impairment in patients undergoing head and neck irradiation. Laryngoscope. 2008;118(1):24-31. doi: https://doi.org/10.1097/MLG.0b013e318155a276

16. Moore C, McLister C, Cardwell C, et al. Dental caries following radiotherapy for head and neck cancer: a systematic review. Oral Oncol. 2020;100:104484. doi: https://doi.org/10.1016/j.oraloncology.2019.104484

17. Ministério da Saúde (BR), Secretaria de Atenção Especializada à Saúde, Departamento de Atenção Hospitalar, Domiciliar e de Urgência. Atenção domiciliar na atenção primária à saúde. Brasília, DF: Ministério da Saúde; 2020.

18. Wang S, Liu Y, Feng Y, et al. A review on curability of cancers: more efforts for novel therapeutic options are needed. Cancers (Basel). 2019;11(11):1782. doi: https:// doi.org/10.3390/cancers11111782

19. Rohr Y, Adams J, Young L. Oral discomfort in palliative care: results of an exploratory study of the experiences of terminally ill patients. Int J Palliat Nurs. 2010;16(9):43944. doi: https://doi.org/10.12968/ijpn.2010.16.9.78638

20. Rinstad T, Bergqvist B, Mattsson U. Follow-up of need for dental treatment in 126 patients who have received radiation treatment to the head and neck region. Int J Dent Hyg. 2020;18(2):201-9. doi: https://doi. org/10.1111/idh.12426

21. Ruiz-Esquide G, Nervi B, Vargas A, et al. Treatment and prevention of cancer treatment related oral mucositis. Rev Med Chil. 2011;139(3):373-81. doi: http://doi. org/10.4067/S0034-98872011000300015

22. Martin L, Senesse P, Gioulbasanis I, et al. Diagnostic criteria for the classification of cancer-associated weight loss. J Clin Oncol. 2015;33(1):90-9. doi: http://doi. org/10.1200/JCO.2014.56.1894

23. Muscaritoli M, Molfino A, Lucia S, et al. Cachexia: a preventable comorbidity of cancer. A T.A.R.G.E.T. approach. Crit Rev Oncol Hematol. 2015;94(2):251-9. doi: http://doi.org/10.1016/j. critrevonc.2014.10.014

24.Zocchi D, Wennemuth G, Oka Y. The cellular mechanism for water detection in the mammalian taste system. Nat Neurosci. 2017;20:927-33. doi: https:/doi. org/10.1038/nn.4575

25. Hovan AJ, Williams PM, Stevenson-Moore P, et al. A systematic review of dysgeusia induced by cancer therapies. Support Care Cancer. 2010;18(8):1081-7. doi: https://doi.org/10.1007/s00520-010-0902-1

26. Pugnaloni S, Vignini A, Borroni F, et al. Modifications of taste sensitivity in cancer patients: a method for the evaluations of dysgeusia. Supportive Care in Cancer. 2020;28(3):1173-81. doi: https://doi.org/10.1007/ s00520-019-04930-x

27. Pinel B, Cassou-Mounat T, Bensadoun RJ. [Oropharyngeal candidiasis and radiotherapy]. Cancer Radiothe. 2012;16(3):222-9. French. doi: https://doi. org/10.1016/j.canrad.2011.11.004 
28. Wilberg P, Hjermstad MJ, Ottesen S, et al. Oral health is an important issue in end-of-life cancer care. Support Care Cancer. 2012;20(12):3115-22. doi: https://doi. org/10.1007/s00520-012-1441-8

29. Astvad K, Johansen HK, Høiby N, et al. Oropharyngeal candidiasis in palliative care patients in Denmark. J Palliat Med. 2015;18(11):940-4. doi: https://doi.org/10.1089/ jpm.2015.29003.ka

30. Bellm LA, Epstein JB, Rose-Ped A, et al. Patient reports of complications of bone marrow transplantation. Support Care Cancer. 2000;8(1):33-9. doi: https://doi. org/10.1007/s005209900095

31. Mercadante S, Aielli F, Adile C, et al. Prevalence of oral mucositis, dry mouth, and dysphagia in advanced cancer patients. Support Care Cancer. 2015;23(11):3249-55. doi: https://doi.org/10.1007/s00520-015-2720-y

32. Peterson DE, Bensadoun RJ, Roila F, et al. Management of oral and gastrointestinal mucositis: ESMO Clinical Practice Guidelines. Ann Oncol. 2011;22(Suppl 6): vi78-84. doi: https://doi.org/10.1093/annonc/mdr391

Recebido em 28/7/2020

Aprovado em 19/11/2020 